\title{
MODELING OF A BINGHAM MODEL OF A MAGNETORHEOLOGICAL DAMPER CONSIDERING STOCHASTIC UNCERTAINTIES IN THEIR GEOMETRIC VARIABLES
}

\author{
Ke Chen, Xiaopeng Yu, Haishuo Wang, Hongmei Zheng, Guojun Zhang, Rui Wu \\ School of Mechanical Engineering, Hefei University of Technology, Hefei, China \\ e-mail: k.chen@163.com; xpyu2013@163.com; hongmeizh@hfut.edu.cn; \\ whs1219@126.com; noqqa6@163.com; wurui8911@163.com
}

\begin{abstract}
Stochastic uncertainty theory is used to develop a new Bingham model of magnetorheological dampers superior to the existing model. Some input variables are defined as stochastic variables by the stochastic factor method, and the stochastic Bingham model is developed by the algebraic synthesis method. Curves of the damping force obtained by the stochastic Bingham model and the Bingham model in the literature are compared with experimental results, revealing that the curves obtained by the stochastic Bingham model are much closer to the experimental curves. Therefore, we confirm that the stochastic Bingham model is superior to the model from the literature.
\end{abstract}

Keywords: Bingham model, literature model, stochastic uncertainty theory, experimental verification

\section{Introduction}

Magnetorheological (MR) dampers have been widely studied because of their controllable damping force, high shear yield limit and low energy consumption (Du et al., 2016). The Ford corporation, which is the most famous magnetorheological fluid (MRF) supplier, first began commercialization of MR dampers (Jia and Zhou, 2009). Stanway et al. (1987) first established a Bingham model in the study of electrorheological dampers. Then, Shames and Cozzarelli (1992) used the Bingham model to study damping force curves of MR dampers. However, hysteretic characteristics and the shear thinning phenomenon were not reflected by the Bingham model. Thus, Wereley et al. (1998) established a dual-viscosity hysteresis model. To study stochastic vibration with hysteresis, Wen established a Bouc-Wen model (Wen, 1976). Then, the Bouc-Wen model was further investigated by a number of researchers (Gan et al., 2016; Zaman and Sikder, 2015). Spencer et al. (1997) developed a phenomenological model based on the Bouc-Wen model to improve the accuracy of the model. Then, Miah et al. (2015) proposed a modified phenomenological model verified by experimental results to describe nonlinear characteristics of rotary MR dampers. Additionally, a rotary MR damper was designed for a vehicle seat and a phenomenological model was utilized to investigate the corresponding dynamic performances (Sun et al., 2016). Aguirre et al. (2012) used a modified Dahl model to study the damping force of large MR dampers, and this model was experimentally validated. A modified Sigmoid model was developed to investigate the damping force of integrated MR dampers. and an experimental validation was conducted (Zhang et al., 2017). Sapiński et al. (2016) developed an energy harvesting MR damper that converted vibration energy into electrical energy. Then, Ahamed et al. (2016) performed a review of energy harvesting MR dampers. Recently, a self-sensing MR damper was developed and experimentally validated (Hu et al., 2017). In terms of vibration control of MR dampers, the fuzzy control theory is generally used (Yang and Cai, 2015; Liem and Ahn, 2016; Tang et al., 2017). 
In the investigations discussed above, geometric variables of MR dampers were only defined as certain variables. However, the geometric variables are uncertain due to kinds of uncertain factors in the engineering. If the geometric variables of MR dampers are defined as uncertain variables, such as stochastic variables, a superior Bingham model of the damping force could be obtained. A stochastic uncertain theory including a stochastic factor method and an algebraic synthesis method was initially developed by Chen working at Xidian University (Chen, 1994). We were the initial researchers to introduce the stochastic uncertain theory including a stochastic factor method and an algebraic synthesis method in an English paper. We made an application of this theory in another paper (Chen et al., 2018). This theory is introduced below.

In this paper, the stochastic uncertainty theory is utilized to investigate the damping force obtained by a Bingham model from the literature under uncertainties of geometric variables. The variation process of the damping force is regarded as a stochastic process. The piston diameter, piston rod diameter, orifice length and orifice diameter are defined as stochastic variables with the stochastic factor method. The stochastic Bingham model is established with the algebraic synthesis method.

\section{Bingham model by Ou (2003)}

An MR damper is mainly composed of several components, including an upper cavity, lower cavity, supplementary room, coil, piston, piston rod, and a damping hole, as shown in Fig. 1.

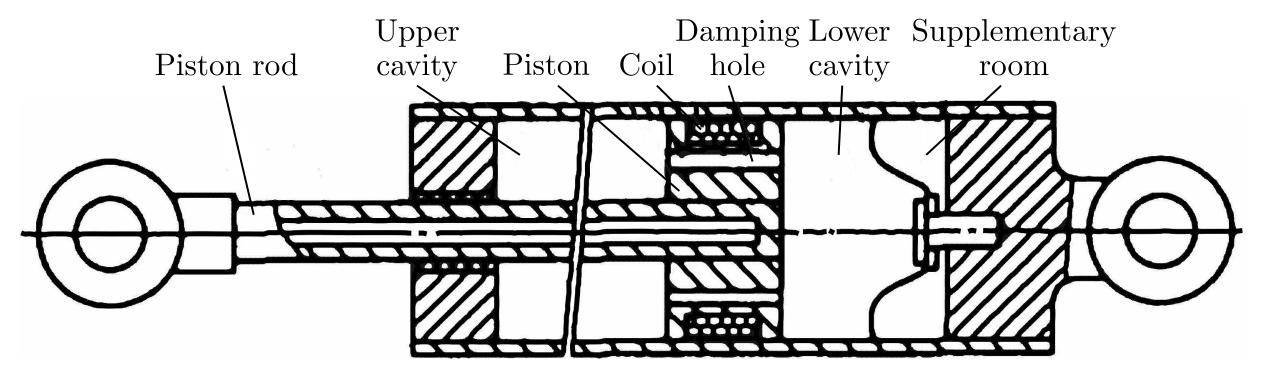

Fig. 1. Structure of the magnetorheological damper (Deng et al., 2008)

The Bingham model of an MR damper is established according to Ou (2003) in the following Sections. The Bingham model is studied under the flow mode.

The shear stress and the shear rate of the MRF are defined as

$$
\tau=\tau_{a} \operatorname{sgn}\left(\dot{v}_{1}\right)+\eta \dot{v}_{1} \quad \dot{v}_{1}=\frac{d v_{1}}{d y}
$$

where $\tau$ is the shear stress of the MRF, $\tau_{a}$ is the shear yield limit of the MRF, $\dot{v}_{1}$ is the shear rate of the MRF, $\eta$ is the dynamic viscosity of the MRF, $v_{1}$ is the speed of the MRF, and $y$ represents the radial direction of the damping hole.

Equation (2.2) is obtained from Eq. (2.1)

$$
\frac{d v_{1}}{d y}=\left\{\begin{array}{lll}
\frac{\tau+\tau_{a}}{\eta} & \text { for } & \tau<-\tau_{a} \\
0 & \text { for } & |\tau| \leqslant \tau_{a} \\
\frac{\tau-\tau_{a}}{\eta} & \text { for } & \tau>\tau_{a}
\end{array}\right.
$$

According to the law of conservation of momentum, Eq. (2.3) is obtained as

$$
\frac{d p}{d x}=\frac{d \tau}{d y}
$$


where $p$ denotes the pressure difference between the two ends of the piston, and $x$ denotes the axial direction. When the value of $y=0$ (the middle position of the damping hole), the value of $\tau=0$.

According to Eq. (2.3), the shear stress $\tau$ is described as

$$
\tau=\frac{d p}{d x} y
$$

Combining Eq. (2.2) and Eq. (2.4), the speed $v_{1}$ is expressed as

$$
v_{1}= \begin{cases}\frac{1}{2 \eta}\left(-\frac{d p}{d x}\right)\left[\left(\frac{h}{2}\right)^{2}-y^{2}\right]-\frac{\tau_{a}}{\eta}\left(\frac{h}{2}-|y|\right) & \text { for } \quad \frac{h_{t}}{2} \leqslant|y| \leqslant \frac{h}{2} \\ \frac{1}{8 \eta\left(-\frac{d p}{d x}\right)}\left[h\left(-\frac{d p}{d x}\right)-2 \tau_{a}\right]^{2} & \text { for } \quad|y| \leqslant \frac{h_{t}}{2}\end{cases}
$$

where $h$ is the diameter of the damping hole.

When the MRF does not reach the yield limit, it only exhibits elastic deformation. When the yield limit is reached, a velocity gradient in the $y$ direction will be generated near the edge of the hole. As shown in Fig. 2, there is no velocity gradient at the $h_{c}$ position, and a velocity gradient appears at the $h_{t}$ position.

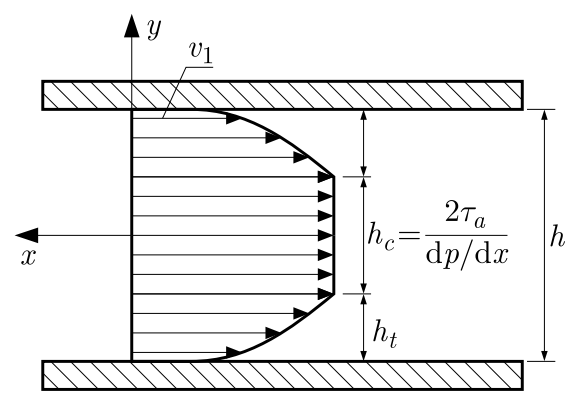

Fig. 2. Velocity distribution of the MRF (Ou, 2003)

The flow of the MRF is defined as

$$
Q=\int_{-h / s}^{h / 2} b v_{1} d y
$$

where $Q$ denotes the flow of the MRF, and $b$ denotes width of the plate perpendicular to the $x y$-plane when the damping hole is assumed to be the gap between the upper and lower plates.

Combining Eq. (2.5) and Eq. (2.6), the flow $Q$ can be described as

$$
Q=\frac{b}{12 \eta}\left(\frac{L}{p}\right)^{2}\left[4 \tau_{a}^{3}-3 \tau_{a} h^{2}\left(\frac{p}{L}\right)^{2}+h^{3}\left(\frac{p}{L}\right)^{3}\right]
$$

where $L$ is length of the damping hole.

Equation (2.8) is obtained from a book by Tao (1997)

$$
p=\frac{12 \eta L Q}{b h^{3}}+\frac{3 L \tau_{a}}{h}
$$

The flow $Q$ can also be defined as

$$
Q=S v_{2}
$$

where $S$ is the effective area of the piston, and $v_{2}$ is the speed of the piston. 
The effective area $S$ is defined as

$$
S=\frac{\pi\left(d_{1}^{2}-d_{2}^{2}\right)}{4}
$$

where $d_{1}$ is the piston diameter, and $d_{2}$ is the piston rod diameter.

The values of $\operatorname{sgn}\left(v_{2}\right)$ are described as

$$
\operatorname{sgn}\left(v_{2}\right)=\left\{\begin{array}{lll}
1 & \text { for } & v_{2}<0 \\
0 & \text { for } & v_{2}=0 \\
-1 & \text { for } & v_{2}>0
\end{array}\right.
$$

Combining Eqs. (2.7)-(2.11), and considering the friction force $F_{f}$ and the opposite direction between the damping force and the piston speed, the Bingham model in Ou (2003) is described as

$$
F_{T}=-\frac{3 \pi \eta L\left(d_{1}^{2}-d_{2}^{2}\right)^{2}}{4 d_{1} h^{3}} v_{2}+\left[\frac{3 \pi L\left(d_{1}^{2}-d_{2}^{2}\right)}{4 h} \tau_{a}+F_{f}\right] \operatorname{sgn}\left(v_{2}\right)
$$

where $F_{T}$ represents the damping force obtained from the Bingham model by Ou (2003).

\section{Stochastic Bingham model of an MR damper}

The stochastic uncertainty theory includes the stochastic factor method, the algebraic synthesis method and some calculation rules. The stochastic factor method and the algebraic synthesis method are only described in Chinese in (Chen, 1994). And the calculation rules are also unsystematic. In this Section, two methods of the stochastic uncertainty theory are described by mathematical formulas, and the calculation rules are expressed clearly.

\subsection{Expected values and mean square errors of the stochastic variables}

The aim of the stochastic factor method is to obtain the expected values and the mean square errors of the stochastic variables. The stochastic factor method is described as

$$
\begin{array}{lr}
A=\bar{A} \widetilde{A} & i(A)=i(\widetilde{A}) \\
E(\widetilde{A})=1 & \sigma(\widetilde{A})=i(\widetilde{A}) E(\widetilde{A}) \\
E(A)=\bar{A} E(\widetilde{A}) & \sigma(A)=i(A) E(A)
\end{array}
$$

where $A$ represents a stochastic variable; $\bar{A}$ and $\widetilde{A}$ represent a certain value and the stochastic factor of $A$, respectively; and $E, \sigma$ and $i$ represent the expected value, the mean square error and the variation coefficient, respectively.

The stochastic uncertain theory including the stochastic factor method and the algebraic synthesis method only researches the mean values and the mean square errors of stochastic variables. $d_{1}, d_{2}, L$ and $h$ are defined as stochastic variables in Eq. (3.2). They do not have certain constants, and their values are of a stochastic distribution. According to the stochastic factor method, the stochastic variables $d_{1}, d_{2}, L$ and $h$ are defined as

$$
d_{1}=\bar{d}_{1} \widetilde{d}_{1} \quad d_{2}=\bar{d}_{2} \widetilde{d}_{2} L=\bar{L} \widetilde{L} \quad h=\bar{h} \widetilde{h}
$$

The expected value of each stochastic factor is

$$
E\left(\widetilde{d}_{1}\right)=1 \quad E\left(\widetilde{d}_{2}\right)=1 \quad E(\widetilde{L})=1 \quad E(\widetilde{h})=1
$$


Thus, the expected value of each stochastic variable is obtained as

$$
\begin{array}{lr}
E\left(d_{1}\right)=\bar{d}_{1} E\left(\widetilde{d}_{1}\right) & E\left(d_{2}\right)=\bar{d}_{2} E\left(\widetilde{d}_{2}\right) \\
E(L)=\bar{L} E(\widetilde{L}) & E(h)=\bar{h} E(\widetilde{h})
\end{array}
$$

The variation coefficient of each stochastic factor is expressed as

$$
i\left(\widetilde{d}_{1}\right)=i\left(d_{1}\right) \quad i\left(\widetilde{d}_{2}\right)=i\left(d_{2}\right) \quad i(\bar{L})=i(L) \quad i(\widetilde{h})=i(h)
$$

The mean square error of each stochastic factor is described as

$$
\begin{array}{lr}
\sigma\left(\widetilde{d}_{1}\right)=i\left(\widetilde{d}_{1}\right) E\left(\widetilde{d}_{1}\right) & \sigma\left(\widetilde{d}_{2}\right)=i\left(\widetilde{d}_{2}\right) E\left(\widetilde{d}_{2}\right) \\
\sigma(\widetilde{L})=i(\widetilde{L}) E(\widetilde{L}) & \sigma(\widetilde{h})=i(\widetilde{h}) E(\widetilde{h})
\end{array}
$$

Therefore, the mean square error of each stochastic variable is expressed as

$$
\sigma\left(d_{1}\right)=\bar{d}_{1} \sigma\left(\widetilde{d}_{1}\right) \quad \sigma\left(d_{2}\right)=\bar{d}_{2} \sigma\left(\widetilde{d}_{2}\right) \quad \sigma(L)=\bar{L} \sigma(\bar{L}) \quad \sigma(h)=\bar{h} \sigma(\widetilde{h})
$$

\subsection{Stochastic Bingham model}

The aim of the algebraic synthesis method is to obtain the expected value and the mean square error of a function including the stochastic variables. The algebraic synthesis method is described as

$$
\begin{aligned}
& Y=f\left(X_{1}, X_{2}, X_{3}, \ldots, X_{n}\right) \\
& E\left(G_{1}\right)=j_{1}\left[E\left(X_{1}\right), \sigma\left(X_{1}\right), E\left(X_{2}\right), \sigma\left(X_{2}\right)\right] \\
& \sigma\left(G_{1}\right)=m_{1}\left[E\left(X_{1}\right), \sigma\left(X_{1}\right), E\left(X_{2}\right), \sigma\left(X_{2}\right)\right] \\
& E\left(G_{2}\right)=j_{2}\left[E\left(G_{1}\right), \sigma\left(G_{1}\right), E\left(X_{3}\right), \sigma\left(X_{3}\right)\right] \\
& \sigma\left(G_{2}\right)=m_{2}\left[E\left(G_{1}\right), \sigma\left(G_{1}\right), E\left(X_{3}\right), \sigma\left(X_{3}\right)\right] \\
& \vdots \\
& E\left(G_{n-1}\right)=j_{n-1}\left[E\left(G_{n-2}\right), \sigma\left(G_{n-2}\right), E\left(X_{n}\right), \sigma\left(X_{n}\right)\right] \\
& \sigma\left(G_{n-1}\right)=m_{n-1}\left[E\left(G_{n-2}\right), \sigma\left(G_{n-2}\right), E\left(X_{n}\right), \sigma\left(X_{n}\right)\right] \\
& E(Y)=E\left(G_{n-1}\right) \\
& \sigma(Y)=\sigma\left(G_{n-1}\right)
\end{aligned}
$$

where $Y$ represents the function, $X_{1}, X_{2}, X_{3}, X_{n}, G_{1}, G_{2}, G_{n-2}, G_{n-1}$ represent the stochastic variables; and $f, j_{1}, m_{1}, j_{2}, m_{2}, j_{n-1}, m_{n-1}$ represent the calculation relationship.

The rules of the mathematical operation among stochastic variables are given in Eqs. (3.9)-(3.14). $A, B$ and $C$ are variables, and $D$ is a constant.

By Taylor expansion, the expected value and the mean square error of $A$ are

$$
A=f(B) \quad E(A)=f[E(B)]+\frac{1}{2} f^{\prime \prime}[E(B)] \sigma^{2}(B) \quad \sigma(A)=f^{\prime}[E(B)] \sigma(B)
$$

The expected value and the mean square error of $A$ are obtained with a second-order and a first-order Taylor series, respectively.

The addition operation is described as

$$
\begin{array}{llrl}
A=B+C & E(A)=E(B)+E(C) & \sigma(A)=\sqrt{\sigma(B)^{2}+\sigma(C)^{2}} \\
A=B+D & E(A)=E(B)+D & \sigma(A)=\sigma(B)
\end{array}
$$


The subtraction operation is expressed as

$$
\begin{array}{llrl}
A=B-C & E(A)=E(B)-E(C) & \sigma(A)=\sqrt{\sigma(B)^{2}+\sigma(C)^{2}} \\
A=B-D & E(A)=E(B)-D & \sigma(A)=\sigma(B)
\end{array}
$$

The multiplication operation is expressed as

$$
\begin{aligned}
& A=B C \quad E(A)=E(B) E(C) \\
& \sigma(A)=\sqrt{[E(B) \sigma(C)]^{2}+[E(C) \sigma(B)]^{2}+[\sigma(B) \sigma(C)]^{2}} \\
& A=D B \quad E(A)=D E(B) \quad \sigma(A)=D \sigma(B)
\end{aligned}
$$

The division operation is described as

$$
\begin{aligned}
& A=\frac{B}{C} \quad E(A)=\frac{E(B)}{E(C)}\left[1+\left(\frac{\sigma(C)}{E(C)}\right)^{2}\right] \\
& \sigma(A)=\frac{E(B)}{E(C)} \sqrt{\left(\frac{\sigma(B)}{E(B)}\right)^{2}+\left(\frac{\sigma(C)}{E(C)}\right)^{2}}
\end{aligned}
$$

The expected value and the mean square error of $A^{2}$ are

$$
E\left(A^{2}\right)=E(A)^{2}+\sigma(A)^{2} \quad \sigma\left(A^{2}\right)=2 E(A) \sigma(A)
$$

The stochastic Bingham model of an MR damper is established by the algebraic synthesis method and the basic expressions in Eqs. (3.9)-(3.14). The derivation process is described in the following expressions.

The expected values and the mean square errors of $d_{1}^{2}$ and $d_{2}^{2}$ are described as

$$
\begin{array}{ll}
E\left(d_{1}^{2}\right)=\left[E\left(d_{1}\right)\right]^{2}+\left[\sigma\left(d_{1}\right)\right]^{2} & \sigma\left(d_{1}^{2}\right)=4\left[E\left(d_{1}\right)\right]^{2}\left[\sigma\left(d_{1}\right)\right]^{2} \\
E\left(d_{2}^{2}\right)=\left[E\left(d_{2}\right)\right]^{2}+\left[\sigma\left(d_{2}\right)\right]^{2} & \sigma\left(d_{2}^{2}\right)=4\left[E\left(d_{2}\right)\right]^{2}\left[\sigma\left(d_{2}\right)\right]^{2}
\end{array}
$$

For ease of calculation, some expressions are replaced by $g_{1}, g_{2}, g_{3}, g_{4}, k_{1}, k_{2}, g_{5}, g_{6}$, which are expressed as

$$
\begin{array}{lrrrr}
g_{1}=d_{1}^{2}-d_{2}^{2} & g_{2}=g_{1}^{2} & g_{3}=L g_{2} & g_{4}=L g_{1} \\
k_{1}=h^{3} & k_{2}=d_{1} k_{1} & g_{5}=\frac{g_{3}}{k_{2}} & g_{6}=\frac{g_{4}}{h}
\end{array}
$$

Therefore, Eq. (2.12) can also be described as

$$
F_{T}=\frac{3 \pi \eta}{4} v_{2} g_{5}+\left(\frac{3 \pi}{4} \tau_{a} g_{6}+F_{f}\right) \operatorname{sgn}\left(v_{2}\right)
$$

The expected values and the mean square errors of $g_{1}, g_{2}, g_{3}, g_{4}$ are expressed as

$$
\begin{array}{lcc}
E\left(g_{1}\right)=E\left(d_{1}^{2}\right)-E\left(d_{2}^{2}\right) & \sigma\left(g_{1}\right)=\sqrt{\left[\sigma\left(d_{1}^{2}\right)\right]^{2}+\left[\sigma\left(d_{2}^{2}\right)\right]^{2}} \\
E\left(g_{2}\right)=\left[E\left(g_{1}\right)\right]^{2}+\left[\sigma\left(g_{1}\right)\right]^{2} & \sigma\left(g_{2}\right)=4\left[E\left(g_{1}\right)\right]^{2}\left[\sigma\left(g_{1}\right)\right]^{2} \\
E\left(g_{3}\right)=E(L) E\left(g_{2}\right) & \sigma\left(g_{3}\right)=\sqrt{\left[E(L) \sigma\left(g_{2}\right)\right]^{2}+\left[E\left(g_{2}\right) \sigma(L)\right]^{2}+\left[\sigma(L) \sigma\left(g_{2}\right)\right]^{2}} \\
E\left(g_{4}\right)=E(L) E\left(g_{1}\right) & \sigma\left(g_{4}\right)=\sqrt{\left[E(L) \sigma\left(g_{1}\right)\right]^{2}+\left[E\left(g_{1}\right) \sigma(L)\right]^{2}+\left[\sigma(L) \sigma\left(g_{1}\right)\right]^{2}}
\end{array}
$$

The expected values and the mean square errors of $k_{1}$ and $k_{2}$ are described as

$$
\begin{aligned}
& E\left(k_{1}\right)=[E(h)]^{3}+3 E(h)[\sigma(h)]^{2} \quad \sigma\left(k_{1}\right)=9[E(h)]^{4}[\sigma(h)]^{2} \\
& E\left(k_{2}\right)=E\left(d_{1}\right) E\left(k_{1}\right) \quad \sigma\left(k_{2}\right)=\sqrt{\left[E\left(d_{1}\right) \sigma\left(k_{1}\right)\right]^{2}+\left[E\left(k_{1}\right) \sigma\left(d_{1}\right)\right]^{2}+\left[\sigma\left(d_{1}\right) \sigma\left(k_{1}\right)\right]^{2}}
\end{aligned}
$$


The expected values and the mean square errors of $g_{5}$ and $g_{6}$ are expressed as

$$
\begin{array}{lrl}
E\left(g_{5}\right)=\frac{E\left(g_{3}\right)}{E\left(k_{2}\right)}\left[1+\left(\frac{\sigma\left(k_{2}\right)}{E\left(k_{2}\right)}\right)^{2}\right] & \sigma\left(g_{5}\right)=\frac{E\left(g_{3}\right)}{E\left(k_{2}\right)} \sqrt{\left(\frac{\sigma\left(g_{3}\right)}{E\left(g_{3}\right)}\right)^{2}+\left(\frac{\sigma\left(k_{2}\right)}{E\left(k_{2}\right)}\right)^{2}} \\
E\left(g_{6}\right)=\frac{E\left(g_{4}\right)}{E(h)}\left[1+\left(\frac{\sigma(h)}{E(h)}\right)^{2}\right] & \sigma\left(g_{6}\right)=\frac{E\left(g_{4}\right)}{E(h)} \sqrt{\left(\frac{\sigma\left(g_{4}\right)}{E\left(g_{4}\right)}\right)^{2}+\left(\frac{\sigma(h)}{E(h)}\right)^{2}}
\end{array}
$$

Therefore, the stochastic Bingham model of an MR damper can be described as

$$
\begin{aligned}
& E\left(F_{S}\right)=-\frac{3 \pi \eta}{4} v_{2} E\left(g_{5}\right)+\left(\frac{3 \pi}{4} \tau_{a} E\left(g_{6}\right)+F_{f}\right) \operatorname{sgn}\left(v_{2}\right) \\
& \sigma\left(F_{S}\right)=\sqrt{\left[-\frac{3 \pi \eta}{4} v_{2} \sigma\left(g_{5}\right)\right]^{2}+\left[\frac{3 \pi}{4} \tau_{a} \sigma\left(g_{6}\right) \operatorname{sgn}\left(v_{2}\right)\right]^{2}}
\end{aligned}
$$

where $E\left(F_{S}\right)$ and $\sigma\left(F_{S}\right)$ denote the expected value and the mean square error of the stochastic damping force $F_{S}$, respectively.

\section{Experimental verification}

\subsection{Values of the variables}

The Bingham model by $\mathrm{Ou}$ and Guan (1999) was the same as that by $\mathrm{Ou}$ (2003), and the corresponding experimental work was primarily performed in Ou and Guan (1999). For comparison with the experimental results, the values of the variables are taken from $\mathrm{Ou}$ and Guan (1999). The external excitation is $A \sin (2 \pi f t)$. The variables and their values are given in Tables 1 and 2. The experiment was performed at Harbin Turbine Company Limited, located in China. The main test equipment was an electro-hydraulic servo material test machine having a $500 \mathrm{kN}$ MTS system. The magnetorheological fluid was developed by Fudan University, located in China. The input voltage was $9 \mathrm{~V}$; the maximum electrical current was $2 \mathrm{~A}$; and the maximum energy consumption was $20 \mathrm{~W}$. The excitation amplitude was $15 \mathrm{~mm}$, and the excitation frequency was $0.5 \mathrm{~Hz}$. Because the reference paper describing the corresponding experimental work only clearly gave the experimental curves (damping force with respect to displacement) under the excitation amplitude of $15 \mathrm{~mm}$ and the excitation frequency of $0.5 \mathrm{~Hz}$. To be compared with the experimental curves, this paper uses the excitation amplitude of $15 \mathrm{~mm}$ and the excitation frequency of $0.5 \mathrm{~Hz}$.

Table 1. Variables and their values, Ou and Guan (1999)

\begin{tabular}{|l|c|}
\hline \multicolumn{1}{|c|}{ Variable } & Value \\
\hline \hline Density $\rho\left[\mathrm{kg} / \mathrm{m}^{3}\right]$ & 9000 \\
\hline Kinematic viscosity $\nu_{o}\left[\mathrm{~m}^{2} / \mathrm{s}\right]$ & $10^{-4}$ \\
\hline Dynamic viscosity $\eta[\mathrm{Pa} \cdot \mathrm{s}]$ & 0.9 \\
\hline Piston diameter $\bar{d}_{1}[\mathrm{~mm}]$ & 100 \\
\hline Piston rod diameter $\bar{d}_{2}[\mathrm{~mm}]$ & 40 \\
\hline Length of damping hole $\bar{L}[\mathrm{~mm}]$ & 40 \\
\hline Diameter of damping hole $h[\mathrm{~mm}]$ & 2 \\
\hline Excitation amplitude $A[\mathrm{~mm}]$ & 15 \\
\hline Excitation frequency $f[\mathrm{~Hz}]$ & 0.5 \\
\hline Excitation cycle $T_{p}[\mathrm{~s}]$ & 2 \\
\hline Friction force $F_{f}[\mathrm{~N}]$ & 180 \\
\hline
\end{tabular}


Table 2. Relationship of $I, B$ and $\tau_{a}$, Ou and Guan (1999)

\begin{tabular}{|c|c|c|}
\hline $\begin{array}{c}\text { Electric current } \\
I[\mathrm{~A}]\end{array}$ & $\begin{array}{c}\text { Magnetic induction } \\
\text { intensity } B[\mathrm{~T}]\end{array}$ & $\begin{array}{c}\text { Shear yield } \\
\text { limit } \tau_{a}[\mathrm{~Pa}]\end{array}$ \\
\hline \hline 0.5 & 0.14 & 3125 \\
\hline 1.0 & 0.19 & 3900 \\
\hline 1.5 & 0.23 & 4350 \\
\hline 2.0 & 0.25 & 5000 \\
\hline
\end{tabular}

\subsection{Results and discussion}

\subsubsection{Identification of the variation coefficient of the stochastic variables}

The variation coefficient of the stochastic variables is a constant. According to professor Chen' research results (Chen, 1994), the parameters having the same type and physical conditions, such as geometrical dimensions, have the same variation coefficient, i.e., $i\left(d_{1}\right)=i\left(d_{2}\right)=i(L)=$ $i(h)=i$. The maximum damping force in the experiment when $I=0.5 \mathrm{~A}$ is applied to identify the variation coefficient of the stochastic variables. According to Ou and Guan (1999), the maximum damping force in the experiment is $1.8667 \mathrm{kN}$ when $I=0.5 \mathrm{~A}$. The variation coefficient ranges from 0.01 to 0.30 . When $I=0.5 \mathrm{~A}$, the expected values of the maximum damping force calculated by the stochastic Bingham model under different variation coefficients are shown in Table 3.

Table 3. Expected values of the maximum damping force under different variation coefficients for $I=0.5 \mathrm{~A}$

\begin{tabular}{|c|c||c|c|}
\hline$i$ & $\max \left[E\left(F_{S}\right)\right][\mathrm{kN}]$ & $i$ & $\max \left[E\left(F_{S}\right)\right][\mathrm{kN}]$ \\
\hline \hline 0.01 & 1.7698 & 0.16 & 1.8343 \\
\hline 0.02 & 1.7705 & 0.17 & 1.8429 \\
\hline 0.03 & 1.7718 & 0.18 & 1.8520 \\
\hline 0.04 & 1.7735 & 0.19 & 1.8617 \\
\hline 0.05 & 1.7758 & 0.20 & 1.8720 \\
\hline 0.06 & 1.7785 & 0.21 & 1.8829 \\
\hline 0.07 & 1.7817 & 0.22 & 1.8944 \\
\hline 0.08 & 1.7855 & 0.23 & 1.9065 \\
\hline 0.09 & 1.7897 & 0.24 & 1.9192 \\
\hline 0.10 & 1.7945 & 0.25 & 1.9326 \\
\hline 0.11 & 1.7998 & 0.26 & 1.9466 \\
\hline 0.12 & 1.8056 & 0.27 & 1.9612 \\
\hline 0.13 & 1.8120 & 0.28 & 1.9765 \\
\hline 0.14 & 1.8189 & 0.29 & 1.9925 \\
\hline 0.15 & 1.8264 & 0.30 & 2.0092 \\
\hline
\end{tabular}

If the expected value of the maximum damping force calculated by the stochastic Bingham model is the closest to the maximum damping force in the experiment, the corresponding variation coefficient is the variation coefficient of the stochastic variables. When $i=0.19$, the expected value is $1.8617 \mathrm{kN}$, which is the closest to the experimental value of $1.8667 \mathrm{kN}$ in Table 3 . Therefore, the variation coefficient of the stochastic variables is 0.19 .

\subsubsection{Experimental verification of the superiority of the model}

According to $\mathrm{Ou}$ and Guan (1999), when the electrical current $I=0.5 \mathrm{~A}, 1.0 \mathrm{~A}, 1.5 \mathrm{~A}$ and $2.0 \mathrm{~A}$, the curves of the damping force obtained in the experiment are shown in Fig. 3. In this 
figure, the innermost curve to the outermost curve corresponds to electrical currents of $0.5 \mathrm{~A}$, 1.0 A, 1.5 A and 2.0 A. For a single curve, it looks irregular, and we only find a symmetrical trend. Here, these curves are only used as standards for comparison. These curves are applied to verify the superiority of the stochastic Bingham model. Therefore, these curves are not analyzed. FE denotes the damping force obtained in the experiment.

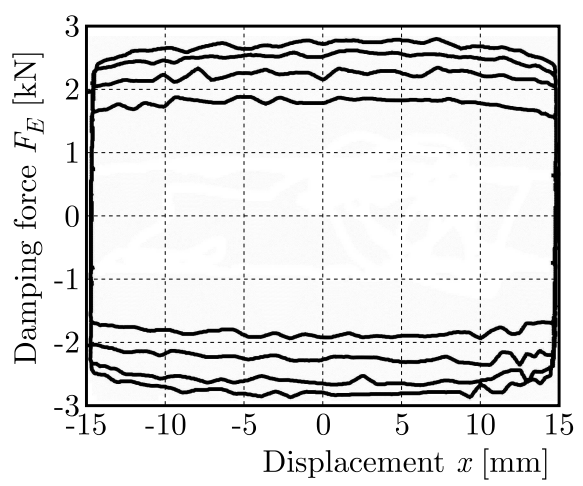

Fig. 3. Variation in the damping force in the experiment with respect to the displacement (Ou and Guan, 1999)

(a)

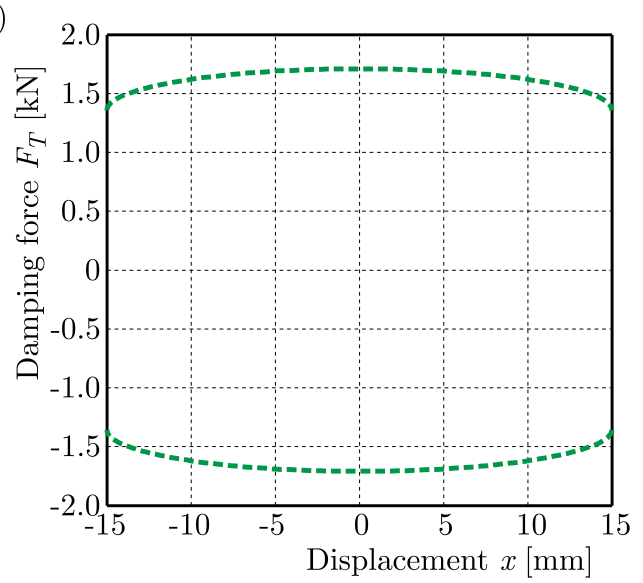

(c)

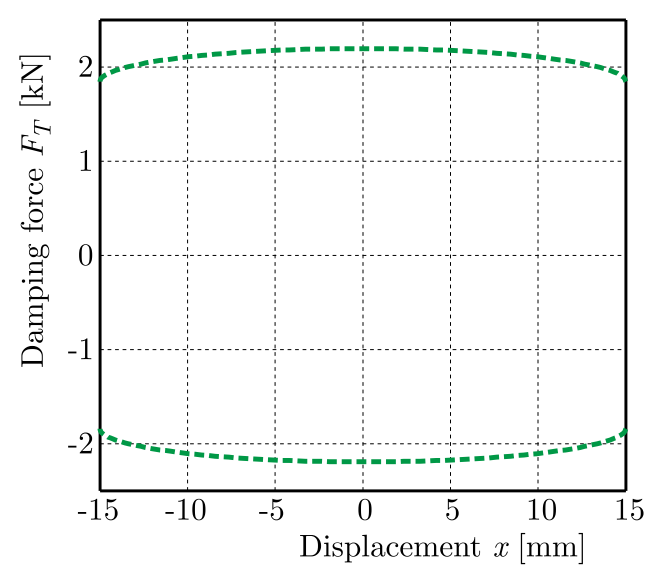

(b)

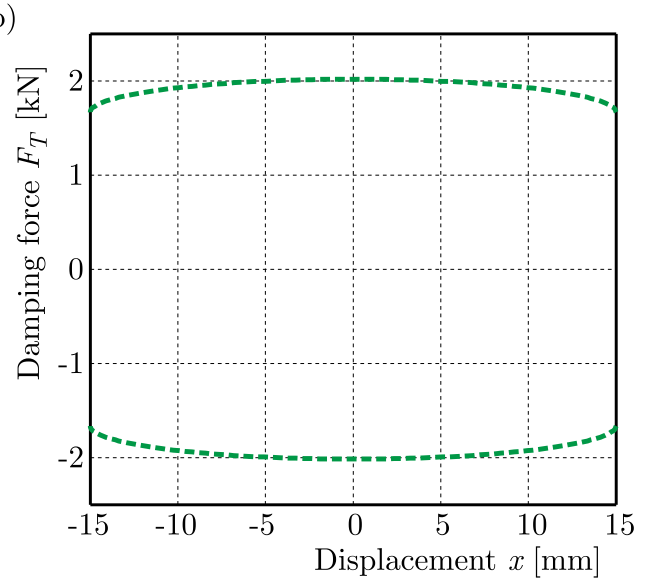

(d)

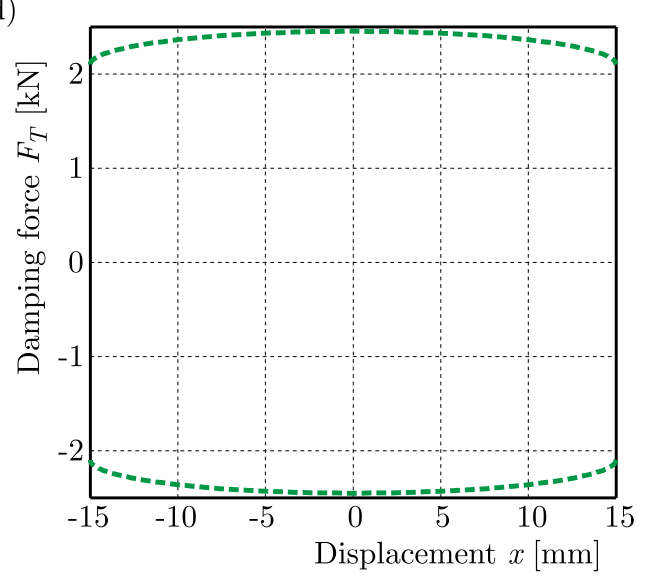

Fig. 4. Variation in the damping force obtained by the Bingham model in $(\mathrm{Ou}, 2003)$ with respect to the displacement; (a) $I=0.5 \mathrm{~A}$, (b) $I=1.0 \mathrm{~A}$, (c) $I=1.5 \mathrm{~A}$, (d) $I=2.0 \mathrm{~A}$

According to the Bingham model established in Section 2, the curves of the damping force are shown in Fig. 4. The curves of the stochastic damping force obtained by the stochastic Bingham 
(a)

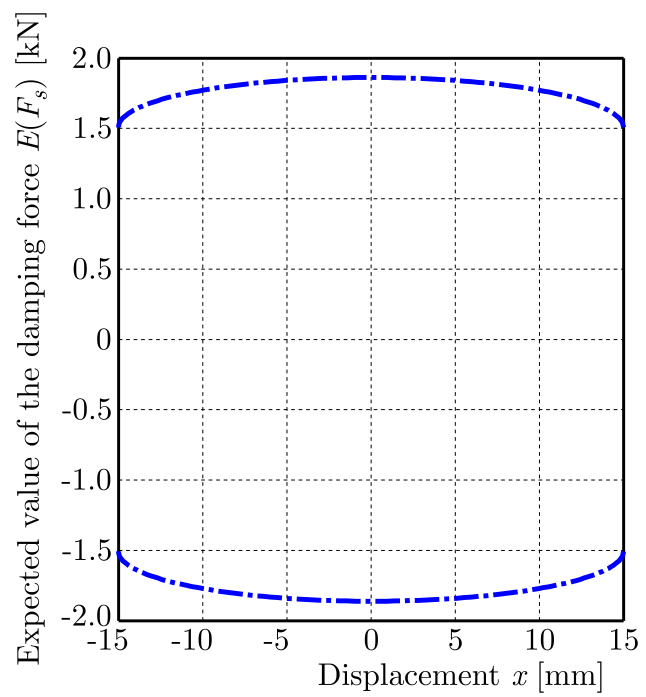

(c)

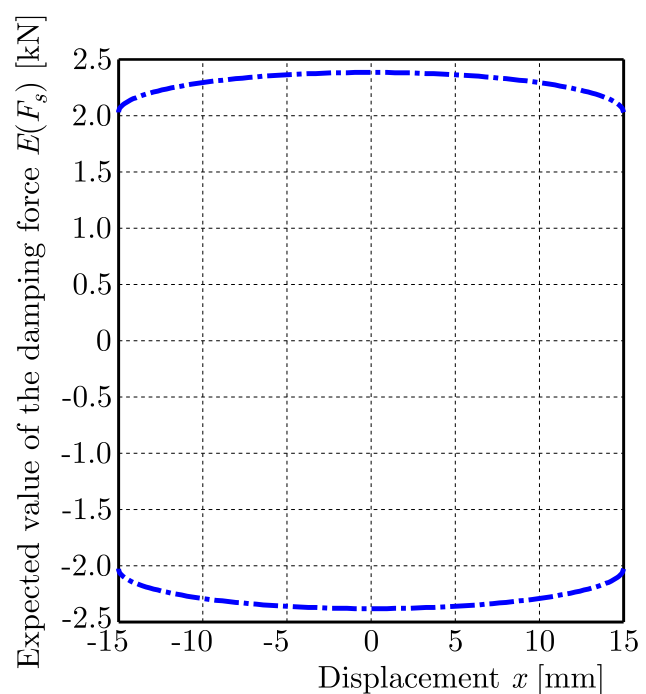

(b)

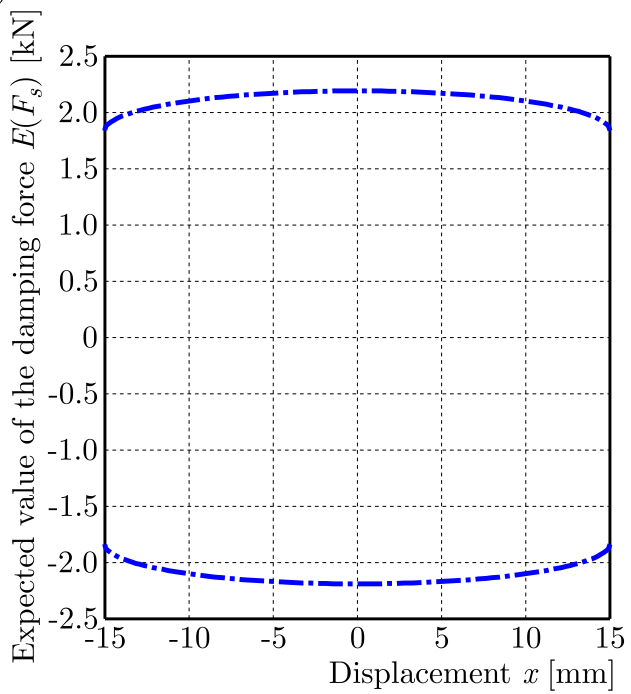

(d)

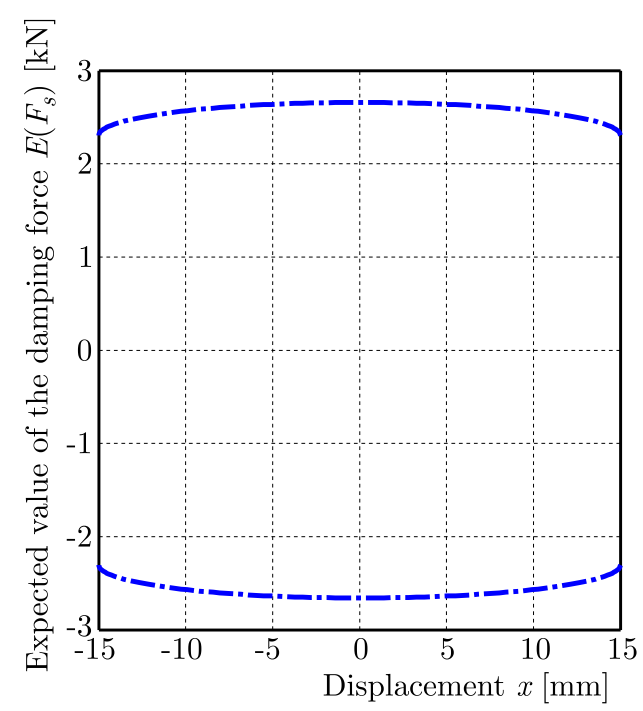

Fig. 5. Variation in the expected value of the damping force obtained by the stochastic Bingham model with respect to the displacement; (a) $I=0.5 \mathrm{~A}$, (b) $I=1.0 \mathrm{~A}$, (c) $I=1.5 \mathrm{~A}$, (d) $I=2.0 \mathrm{~A}$

model established in Section 3 are shown in Fig. 5. Figures 4 and 5 show that the damping force increases with an increase in the electrical current. The reason for this phenomenon is that the mechanical energy providing the damping force increases with the increasing magnetic energy, which is generated by electrical energy. Because of periodic motion of the piston, the variation curves of the damping force are symmetrical.

For ease of comparison among the curves obtained in the experiment, the Bingham model by $\mathrm{Ou}(2003)$ and the stochastic Bingham model, the curves in Figs. 3, 4 and 5 are placed in Fig. 6. The curves obtained in the experiment can be used as standards for comparison.

Figure 6 compares the damping force curves obtained from the stochastic Bingham model and the Bingham model by Ou (2003) with the damping force curves obtained in the experiment when the electrical current $I=0.5 \mathrm{~A}, 1.0 \mathrm{~A}, 1.5 \mathrm{~A}$ and $2.0 \mathrm{~A}$. The damping force curves obtained by the stochastic Bingham model are closer to the experimental curves than the curves obtained by $\mathrm{Ou}$ (2003), indicating that the stochastic Bingham model is much more realistic than $\mathrm{Ou}$ (2003) model under the physical conditions described in Section 4.1. Therefore, the stochastic Bingham model is superior to the Bingham model by Ou (2003) under the physical conditions described in Section 4.1. 
(a)

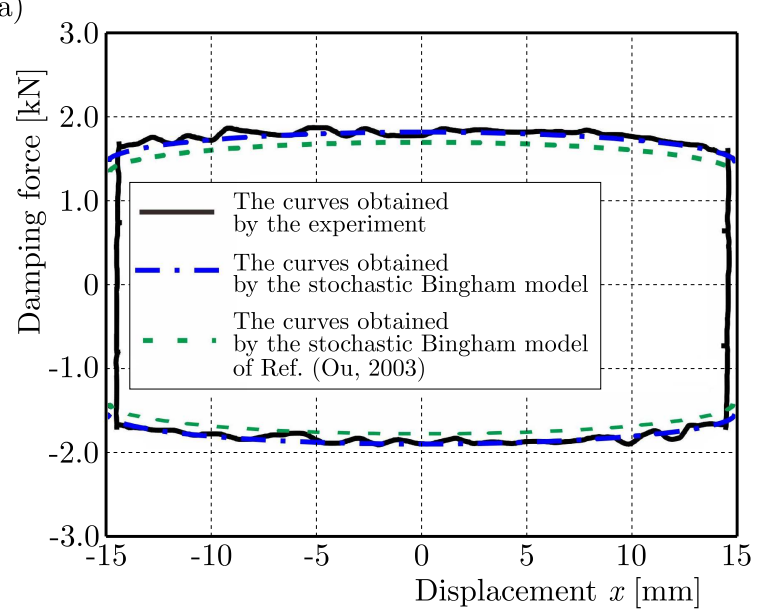

(c)

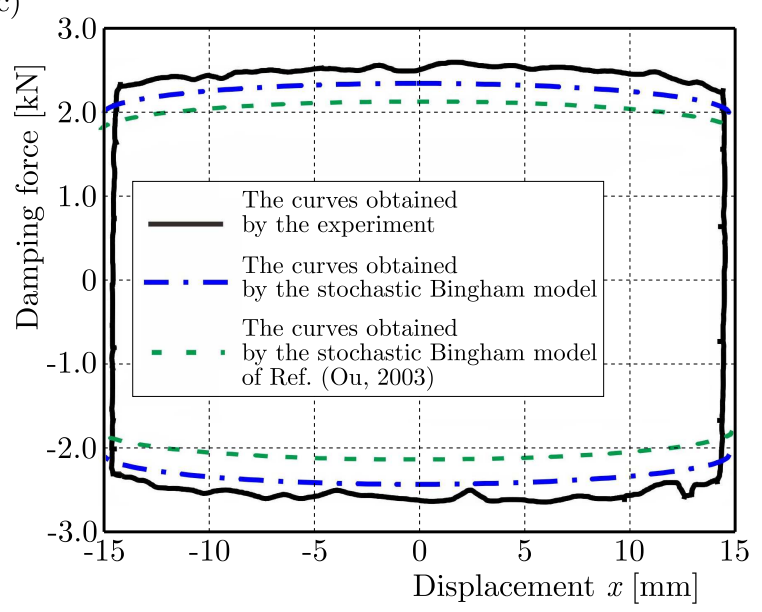

(b)

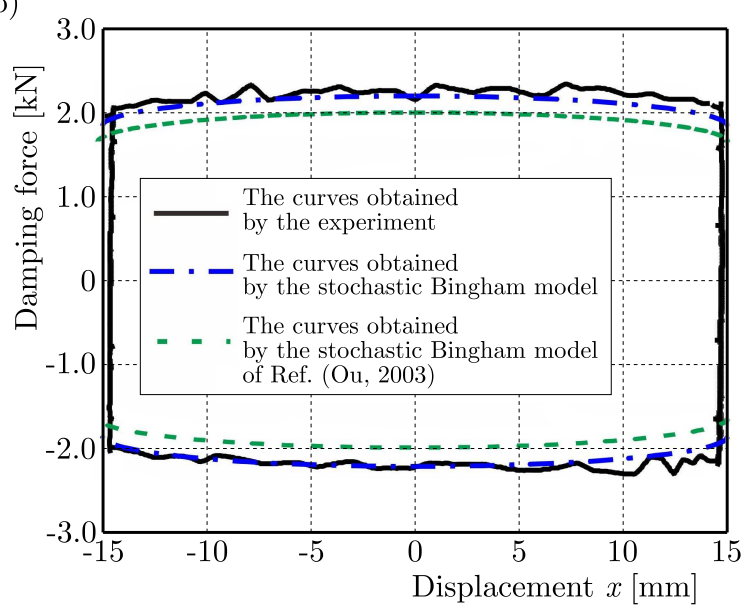

(d)

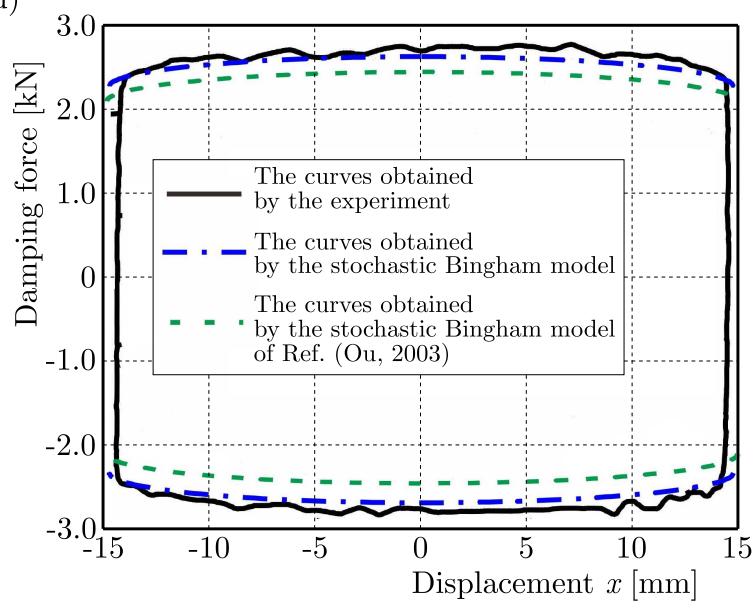

Fig. 6. Variation curves of the damping force from the experiment, the stochastic Bingham model and the Bingham model in $(\mathrm{Ou}, 2003)$; (a) $I=0.5 \mathrm{~A}$, (b) $I=1.0 \mathrm{~A}$, (c) $I=1.5 \mathrm{~A}$, (d) $I=2.0 \mathrm{~A}$

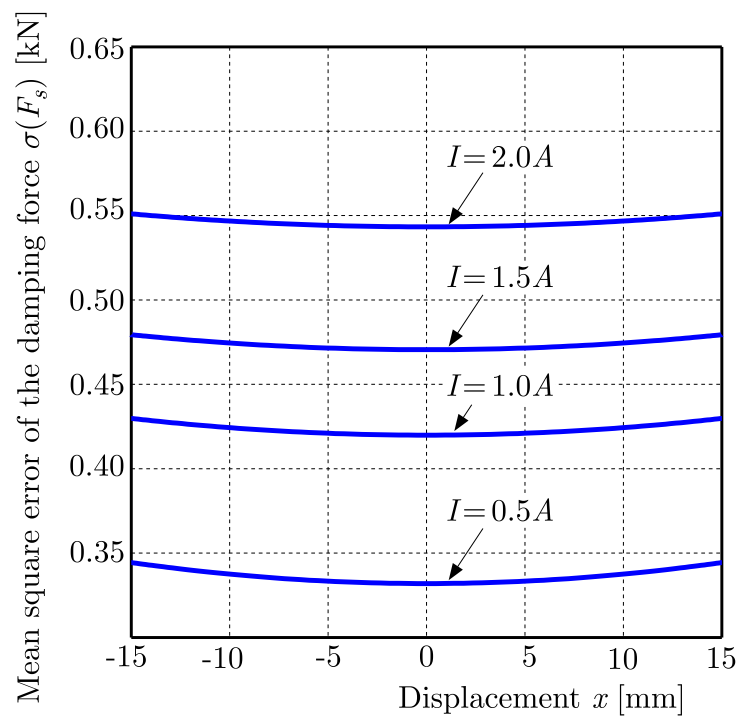

Fig. 7. Variation in the mean square error of the damping force obtained by the stochastic Bingham model with respect to the displacement 
The variation curves of the mean square error for the damping force are also obtained by the stochastic Bingham model established in Section 3, as shown in Fig. 7. The results show that the mean square error of the damping force increases with the increasing electric current. This phenomenon indicates that the mean value of the distances between the damping forces and the mean damping force increases with the increasing electrical current. In comparison with Fig. 5, we find that the mean square error of the damping force increases with the decreasing absolute value of the expected value of the damping force. This phenomenon reveals a relationship between the mean square error and the expected value for the damping force.

\section{Conclusions}

In this paper, a stochastic Bingham model of an MR damper is established by the stochastic uncertainty theory. The piston diameter, piston rod diameter, orifice diameter and orifice length are defined as stochastic variables by the stochastic factor method. The stochastic Bingham model is established by the algebraic synthesis method. The following conclusions are drawn:

- The variation coefficient of the stochastic variables is identified by an experimental result. And it is 0.19 .

- Superiority of the stochastic Bingham model is verified with experimental results under physical conditions described in Section 4.1. The damping force curves obtained by the stochastic Bingham model and the Bingham model by Ou (2003) are compared with the experimental curves respectively. The damping force curves obtained by the stochastic Bingham model are closer to the damping force curves in the experiment than those obtained by $\mathrm{Ou}$ (2003), indicating that the stochastic Bingham model is superior to the Bingham model by Ou (2003) under the physical conditions described in Section 4.1.

- Some rules of the variation curves of the expected value and the mean square error for the damping force are also revealed.

\section{References}

1. Ahamed R., Ferdaus M.M., Li Y.C., 2016, Advancement in energy harvesting magneto-rheological fluid damper: a review, Korea-Australia Rheology Journal, 28, 355-379

2. Aguirre N., Ikhounne F., Rodellar J., Christenson R., 2012, Parametric identification of the Dahl model for large scale MR dampers, Structural Control and Health Monitoring, 19, 332-347

3. Chen J.J., 1994, Reliability of Mechanical and Structural Systems (in Chinese), 1st ed., Xi-Dian University Press, Xi'an

4. Chen K., Yu X.P., Zheng H.M., Wang Y.Y., Zhang G.J., Wu R., 2018, Modeling dissipative heating of hydraulic dampers under consideration of stochastic uncertainties in their geometric parameters, Journal of the Brazilian Society of Mechanical Sciences and Engineering, 40, 312

5. Deng Z.D., Gao F., Liu X.D., Xu G.Y., 2008, Theoretical calculation and testing of damping characteristics for magnetorheological damper on vehicle (in Chinese), Chinese Journal of Mechanical Engineering, 44, 202-207

6. Du X.P., Chen H., Liu Z.J., Wang C., 2016, Semi-active control of space manipulator soft contacting based on magnetorheological rotational damper, Proceedings of the Institution of Mechanical Engineers, Part C: Journal of Mechanical Engineering Science, 230, 2390-2398

7. Gan M.G., Qiao Z., Li Y.L., 2016, Sliding mode control with perturbation estimation and hysteresis compensator based on Bouc-Wen model in tackling fast-varying sinusoidal position control of a piezoelectric actuator, Journal of Systems Science and Complexity, 29, 367-381 
8. Hu G. L., Lu Y., Sun S.S., Li W.H., 2017, Development of a self-sensing magnetorheological damper with magnets in-line coil mechanism, Sensors and Actuators A: Physical, 255, 71-78

9. JiA Y.S., Zhou K.K., 2009, Rheological properties analysis and experiment of magnetorheological fluid for automobile (in Chinese), Journal of Mechanical Engineering, 45, 246-250

10. Liem D.T., Ahn K.K., 2016, Adaptive semi-parallel position/force-sensorless control of electrohydraulic actuator system using MR fluid damper, International Journal of Precision Engineering and Manufacturing, 17, 1451-1463

11. Мiah M.S., Chatzi E.N., Dertimanis V.K., Weber F., 2015, Nonlinear modeling of a rotational MR damper via an enhanced Bouc-Wen model, Smart Materials and Structures, 24, 105020

12. Ou J.P., 2003, Structural Vibration Control-Active, Semi-Active and Intelligent Control (in Chinese), 1st ed., Science Press, Beijing

13. Ou J.P., GuAN X.C., 1999, Experiment study of magnetorheological damper performance, Earthquake Engineering and Engineering Vibration (in Chinese), 1st ed., Science Press, Beijing

14. Sapiński B., RosóŁ M., Węgrzynowski M., 2016, Investigation of an energy harvesting MR damper in a vibration control system, Smart Materials and Structures, 25, 125017

15. Shames I.H., Cozzarelli F.A., 1992, Elastic and Inelastic Stress Analysis, 1st ed., Englewood Cliffs: Prentice Hall, Englewood

16. Spencer B.F., Dyke S.J., Sain M.K., Carlson J.D., 1997, Phenomenological model of a magnetorheological damper, Journal of Engineering Mechanics, 123, 230-238

17. Stanway R., Sproston J.L., Stevens N.G., 1987, Non-linear modeling of an electro-rheological vibration damper, Journal of Electrostatics, 20, 167-184

18. Sun S.S., Ning D.H., Yang J., Du H., Zhang S.W., Li W.H., 2016, A seat suspension with a rotary magnetorheological damper for heavy duty vehicles, Smart Materials and Structures, 25, 105032

19. Tang X., Du H.P., Sun S.S., D. Ning H., Xing Z.W., Li W.H., 2017, Takagi-Sugeno fuzzy control for semi-active vehicle suspension with a magnetorheological damper and experimental validation, IEEE/ASME Transactions on Mechatronics, 22, 291-300

20. TAо B.Q., 1997, Structure of Intelligent Material (in Chinese), 1st ed., National Defense Industry Press, Beijing

21. WEN Y.K., 1976, Method for random vibration of hysteretic systems, Journal of the Engineering Mechanics Division, 102, 249-263

22. Wereley N.M., Pang L., Kamath G.M., 1998, Idealized hysteresis modeling of electrorheological and magnetorheological damper, Journal of Intelligent Materials Systems and Structures, 9, 642-649

23. Yang M.G., CAi C.S., 2015, Longitudinal vibration control for a suspension bridge subjected to vehicle braking forces and earthquake excitations based on magnetorheological dampers, Journal of Vibration and Control, 22, 1-20

24. Zaman M.A., Sikder U., 2015, Bouc-Wen hysteresis model identification using modified firefly algorithm, Journal of Magnetism and Magnetic Materials, 395, 229-233

25. Zhang X.C., Zhang X., Zhao Y.X., Zhao J., Xu Z.D., 2017, Experimental and numerical studies on a composite MR damper considering magnetic saturation effect, Engineering Structures, 132, 576-585 